\title{
XIX. Neue Minerale aus Chile, ein neues Vorkommen von Utahit und ein neues Wismuthcarbonat von Schneeberg.
}

\author{
Von \\ A. Arzruni ( $t$ ) und K. Thaddéeff in Aachen, vollendet und herausgegeben \\ von A. Dannenberg. \\ (Aus dem mineralogischen Institute der königl. technischen Hochschule.)
}

\section{Vorbemerkung des Herausgebers.}

Die Untersuchung der hier beschriebenen neuen Minerale war von Prof. Arzruni schon vor mehreren Jahren begonnen und, dem Eintreffen immer neuer Sendungen chilenischer Minerale folgend, bis zum Sommer 1896 fortgesetzt worden. Verschiedene störende Verhältnisse, die Uebersiedelung des mineralogischen Institutes in ein neues Gebäude, namentlich aber Prof. Arzruni's andauernde Krankheit und dadurch verursachte Abwesenheit von Aachen verzögerten die Veröffentlichung der Ergebnisse. Erst im Sommer vorigen Jahres (1898) konnte Prof. Arzru ni daran denken, diese abschliessend zusammen zu stellen. Ein allzu früher Tod riss ihn mitten aus dieser und anderen wissenschaftlichen Arbeiten, welche ihn trotz schweren Leidens bis zu seinem Ende beschäftigten.

Im Nachlasse fanden sich die Messungen und Berechnungen der drei hier beschriebenen neuen Minerale, sowie die Beobachtung über deren optisches Verhalten, ferner auch der Entwurf einer Einleitung, welcher im Folgenden benutzt wurde.

Die drei neuen Minerale aus Chile erhalten die Namen Arzrunit, Stelznerit, Rafaelit.

Hierzu ist zu bemerken, dass der Name Stelznerit dem betreffenden Minerale von Prof. Arzruni selbst beigelegt ist als eine dem Andenken des verewigten, von ihm hochverehrten Forschers und speciell auch seinen Verdiensten um die Mineralogie Chiles dargebrachte Huldigung. Dass ich die Benennung eines weiteren Minerales an den Namen meines unvergesslichen 
Lehrers lnn̈̈pfe, bedarf sicher keiner Rechtfertigung. Der dritte Name wurde von dem Fundorte des betreffenden Minerales: Mina San Rafael, abgeleitet. Für das krystallographisch nicht genügend charakterisirte Wismuthcarbonat wurde von einer besonderen Benennung abgesehen. Die chemischen Untersuchungen sind von Herrn Thaddéeff ausgeführt und vollständig zusammengestellt.

Aachen, im Januar 1899.

A. Dannenberg.

Seit dem Jahre 1893 beehrte Herr Ingenieur Theodor Hohmann ${ }^{1}$ ) das mineralogische Institut der hiesigen kgl. Technischen Hochschule mit Zusendungen chilenischer und bolivianischer Minerale. Unter ersteren sind namentlich Sulfate und Chloride des Kupfers und Bleies und Sulfate des Eisens bemerkenswerth, von denen manche nicht nur überhaupt, sondern auch aus Chile schon bekannt und beschrieben sind. So sind, um nur einige zu nennen, in den Sendungen vertreten: Linarit, Brochantit, Atacamit, Schwartzembergit, Blödit, Darapskit, Hohmannit, Copiapit, Ferronatrit, Sideronatrit, Caracolit, Daviesit, Percylith, Anglesit, Cerussit u. a. Indessen auch neue Minerale haben sich feststellen lassen. Es wurden drei solche näher untersucht; die Ergebnisse der an ihnen angestellten Beobachtungen sollen im Nachstehenden wiedergegeben werden.

\section{Arzrunit.}

Das Mineral, wie die Analyse zeigt ein Doppelsalz und zwar eine Verbindung von basischem Bleisulfat mit basischem Kupferchlorid, bildet drusige krystallinische Ueberzüge in Höhlungen des zellig porösen, wie zerfressen aussehenden, vorwiegend quarzigen Ganggesteins. Die Farbe dieser Krystallkrusten ist ein lebhaftes Blau oder genauer Blaugrün, entsprechend Nr. 18 ("Blaugrün, zweiter Uebergang nach Blau *) der Radd e* schen Farbenscala, und bewegt sich hier, je nach der Dicke der Krusten, etwa in den Nüancen $g-k$.

Selbständige, gut ausgebildete Krystalle sind verhältnissmässig selten; es gelingt nur schwer und immer nur unvollkommen, solche aus der innigen Verwachsung mit ihrer krystallinen Unterlage abzulösen. Dieser Umstand erschwert etwas die krystallographische und optische Untersuchung.

Die Länge der grössten Kryställchen erreicht kaum $1 \mathrm{~mm}$, ihre Dicke beträgt etwa $\frac{1}{2}$ der Länge. Der Habitus ist hexagonal, entsprechend der Combination eines vorherrschenden Prismas mit Basis und schmaler Abstumpfung der Combinationskanten durch eine Pyramide. Auch die Messung bestätigt scheinbar die hexagonale Form. Trotzdem muss der Arzrunit als rhombisch angesehen werden, wie sein optisches Verhalten beweist.

1) Inzwischen ebenfalls verstorben. Der Herausg. 
Zunächst zeigt sich ein sehr starker Pleochroïsmus nach den verschiedenen Flächen des scheinbaren hexagonalen Prismas: während die Kryställchen beim Durchsehen durch ein Flächenpaar dieser Zone tief blau erscheinen, werden sie nach einem anderen Flüchenpaar derselben Zone gesehen fast farblos oder höchstens noch ganz schwach bläulich gefärbt. Die blaue Färbung ist im ersteren Falle allerdings nicht ganz gleichmässig, sondern oft stellenweise etwas heller und dann stärker ins Grünlichblaue ziehend.

Im convergenten Lichte beobachtet man auf den im durchgehenden Lichte farblos erscheinenden Flächen den nahezu senkrechten Austritt einer optischen Axe.

Die Auslöschung zwischen gekreuzten Nicols ist natürlich gerade, $d . h$. $\|$ und $\perp$ den Prismenkanten.

Es ergiebt sich für die nach Vorstehendem als rhombisch aufzufassenden Krystalle des Arzrunits die Combination:

$$
\{110\},\{010\},\{111\},\{021\},\{001\} \text {. }
$$

Die Messung weist in der Prismenzone keinen Unterschied der Winkel $(110):(1 T 0)$ und $(110):(010)$ nach. Die Wer'he schwanken regellos um $60^{\circ}$ herum $\left(59^{0} 26^{\prime}\right.$ bis $\left.60^{0} 14^{\prime}\right)$. Trotz der theoretisch anzunehmenden Verschiedenheit muss man daher bis auf Weiteres beide Winkel praktisch gleich setzen $\left(60^{\circ}\right)$, mit anderen Worten ein rhombisches Prisma von (sehr nahezu) $120^{\circ}$ annehmen. Ebensowenig erlauben die Messungen eine sichere Unterscheidung von Pyramide und Brachydoma. Es ergeben sich dann folgende Werthe:

$$
\begin{aligned}
& \text { Mittel: } \\
& \left.\begin{array}{l}
(110):(1 T 0) \\
(110):(010)
\end{array}\right\} 60^{\circ} 0^{\prime} \\
& (110):(001) \quad 90 \quad 0 \\
& \left.\begin{array}{l}
(110):(111) \\
(010):(021)
\end{array}\right\} 5028 \\
& \text { (111):(1T1) } 3728 \\
& (111):(001)\} 3937 \\
& a: b: c=0,5773: 1: 0,4163 .
\end{aligned}
$$

Farblose, rhombische Kryställchen auf denselben Stufen gehören wahrscheinlich dem Daviesit an. Sie werden begrenzt von den Flächen des Makropinakoids, einer Pyramide und einem Prisma. Der Habitus ist breit tafelförmig nach $\{100\}$ und gestreckt nach der Verticalaxe. Die wichtigsten der gemessenen Winkel sind: 
2:32 A. Alz'uni ( $t$ ) und $K$. Thaddeelf, vollendet und herausgeg. von $A$. Dannenberg.

Flächen: Symbol:

$a: m=(100):(110)=51013^{\prime}-53017,5^{\prime}$

$m: m=(110):(110) \quad 7416,5-7649$

$\mathrm{t}: \mathrm{t}=(121):(121)$

$\begin{array}{llll}33 & 13-33 & 5 \%\end{array}$
Daviesit (Fle t cher)

berechnet: gemessen:

Fundort: Mina Buena Esperanza, Challacollo, Provinz Tarapaca, Chile.

\section{Stelznerit, ein neues basisches Kupfersulfat.}

Der Stelznerit bildet schön grüne, durchscheinende, prismatische Kryställchen von lebhaftem Glanze, welche auf einer gleichartigen, nur etwas heller grünen, krystallinischen und ziemlich lockeren Masse aufsitzen. Im Aussehen erinnert das Mineral zunächst an Brochantit, dem es, wie die Analyse zeigt, auch chemisch nahe steht.

Dem optischen Verhalten nach kennzeichnet sich der Stelznerit zunächst durch deutlichen Pleochroïsmus. Die prismatischen Krystalle erscheinen blaugrün ( $16 \mathrm{~h}-\mathrm{m}$ der Radde'schen Scala), bei Schwingungen parallel der Prismenkante dagegen mehr gelblichgrün $(15 \mathrm{~h}-\mathrm{i})$ in der dazu senkrechten Richtung.

Die Ebene der optischen Axen liegt $\|\{001\}$ (beim Brochantit parallel dem Orthopinakoid); auf den Prismenflächen tritt je eine optische Axe fast senkrecht aus.

Die Krystalle gehören dem rhombischen Systeme an. Sie werden gebildet von der Combination eines Prismas $(m)$ mit einem Pinakoid $(b)$, einer Pyramide $(p)$, ferner einem dem Pinakoide entsprechenden Doma $(o)$ und der Basis $(c)$. Wird die Pyramide als Grundform genommen, so ergeben sich die Symbole der einzelnen Flächen: $\{111\},\{110\},\{010\},\{011\},\{001\}$.

Die Messungen ergaben folgende Werthe:

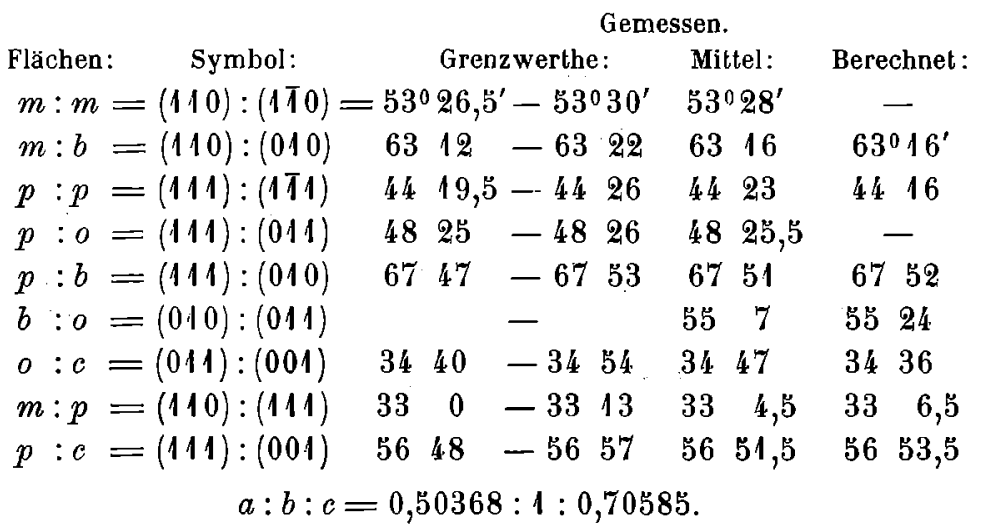

Fundort: Remolinos, Vallinar, Chile. 


\section{Rafaëlit, ein neues Bleioxychlorid.}

Die durchscheinenden, violettrothen Krystallnädelchen dieses Minerals (ca. $0,5-1 \mathrm{~mm}$ lang und $0,2-0,5 \mathrm{~mm}$ breit) bilden einen krustenartigen Ueberzug auf einer Unterlage von Bleiglanz, Quarz, Cölestin. Daneben erscheint noch ein gelbes, theilweise krystallisirtes Mineral, das nach Löthrohrverhalten etc. als Schwartzembergit bestimmt werden konnte; einzelne in diesem Gemenge auftretende blaugrüne Flecken dürften dem Arzrunit angehören, sind jedoch nicht sicher bestimmbar.

Optische Eigenschaften. Pleochroïsmus stark, und zwar tief violett für Schwingungen in der Längsrichtung der Nädelchen und violettroth senkrecht dazu. Sehr dünne Krystalle erscheinen in letzterer Stellung nur noch blass röthlichgelb. Die Färbung ist oft keine gleichmässige, sondern schichtenweise verschieden stark; die Farbenvertheilung ist dann eine zonare, parallel der äusseren Begrenzung des Krystalls. Je nach der Intensität der Färbung hat man

parallel der Längsrichtung: ungefähr $22 \mathrm{i}$ und $23 \mathrm{k}$, senkrecht dazu: etwa 15h-i

der Radde'schen Scala.

Auf der Fläche $a(100)$ sieht man im convergenten Lichte ein doppeltes Axenbild: eine $\Lambda$ xe mit weiteren und eine mit engeren isochromatischen Curven. Dies deutet auf eine Zwillingsbildung nach (100), welche auch durch die Messung (s. u.) nachgewiesen wird.

Die Auslöschung der Krystallnadeln erfolgt parallel und senkrecht zur Längsrichtung.

Glan z: Metallartiger Diamantglanz.

Krystallsystem: Monoklin.

Die Krystalle sind nach der Orthodiagonale gestreckt und in dieser Zone besonders flächenreich. Gut entwickelt ist auch die Verticalzone, in welcher ausser den beiden Pinakoiden noch zwei Prismen auftreten. Untergeordnet erscheinen dann noch zwei Pyramiden. Es wurden insgesammt folgende Formen beobachtet:

1) Orthodiagonale Zone:

2) Verticalzone :

$$
\begin{gathered}
a\{100\}, c\{001\}, d\left\{T_{01}\right\}, l\left\{\bar{T}_{02}\right\}, e\{\overline{\mathbf{2}} 01\}, f\{\overline{3} 01\}, r\{\bar{T} 03\}, \\
h\{101\}, q\{201\} .
\end{gathered}
$$

$$
a\{100\}, m\{110\}, \approx\{130\}, b\{010\} .
$$

3) Pyramiden:

$$
x\{132\}, y\{432\} \text {. }
$$

Die entsprechenden Winkelwerthe sind: 
234 A. Arzruni ( + ) und K. Thaddéeff, vollendet und herausgeg. von A. Dannenl,erg.

\begin{tabular}{|c|c|c|c|c|}
\hline \multirow{3}{*}{$\begin{array}{c}\text { Flächen: } \quad \text { Symbole: } \\
a: c=(100):(001)\end{array}$} & \multicolumn{4}{|c|}{ Gemessen. } \\
\hline & \multicolumn{2}{|c|}{ Grenzwerthe: } & \multirow{2}{*}{$\begin{array}{l}\text { Miitel: } \\
62^{0} 47^{\prime}\end{array}$} & \multirow{2}{*}{ Berechnel: } \\
\hline & $62^{0} 38^{\prime}$ & $-62^{0} 52^{\prime}$ & & \\
\hline$d: c=(\overline{1} 01):(001)$ & 7135 & $-71 \quad 49$ & 714.5 & - \\
\hline$a: e=-(100):(\overline{2} 01)$ & 2155 & -2158 & 2157 & 21056,5 \\
\hline$e: d=(\overline{2} 01):(\bar{T} 01)$ & 2323 & -2331 & 2329 & $23 \quad 31,5$ \\
\hline$a: d=(100):(\pi 01)$ & 4527 & -4532 & 4.530 & 4528 \\
\hline$c: l==(001):($ T02 $)$ & 4025 & -4028 & 4027 & 4026 \\
\hline$l: d==(\bar{T} 02):(\bar{T} 01)$ & 3120,0 & -3121 & 3121 & $31 \quad 19$ \\
\hline$e: f=(\overline{2} 01):(\overline{3} 01)$ & & - & 751 & $7 \check{5} 0,5$ \\
\hline$f: a=(\overline{3} 01):(\bar{\top} 00)$ & & - & 147 & $14 \quad 6$ \\
\hline$c: r=(001):(\overline{1} 03)$ & & - & $26 \quad 19$ & 2622 \\
\hline$r: d=(T 03):(T 01)$ & & - & 4528 & 4033 \\
\hline$h: a=(101):(100)$ & & - & $26 \quad 28$ & 2625,5 \\
\hline$h: c=(101):(001)$ & & 一 & $36 \quad 22$ & 3621,5 \\
\hline$q: a=(201):(100)$ & & - & $15 \quad 54$ & 1554 \\
\hline$q: c=(201):(001)$ & & - & 4652 & 4653 \\
\hline$a: z=(100):(130)$ & 6727 & -6732 & 6728 & - \\
\hline$z: m=(130):(110)$ & $28 \quad 41$ & -2845 & $28 \quad 43$ & 2841 \\
\hline$m: a=(110):(100)$ & $\begin{array}{ll}38 & 43\end{array}$ & -3851 & $38 \quad 46$ & 3847 \\
\hline$z: b=(130):(010)$ & 2232 & -2233 & 2232,5 & 2232 \\
\hline$z: z=(130):(\overline{1} 30)$ & $45 \quad 3$ & $-45 \quad 5$ & 45 & 454 \\
\hline$z: c=(130):(001)$ & 7951 & $-79 \check{5} 9$ & 7955 & 7954,5 \\
\hline$a: x=(100):(132)$ & 5830 & -5834 & $58 \quad 32$ & 5828 \\
\hline$x: y=(132):(432)$ & & - & $\stackrel{2}{8} 85$ & 281 \\
\hline$a: y=(100):(432)$ & & - & 3029 & 3027 \\
\hline$c: x=(001):(132)$ & & - & $52 \quad 40$ & 5237 \\
\hline$x: z=(132):(130)$ & & - & 2711 & 2717,5 \\
\hline An Zwilling nach $\{10$ & & & & \\
\hline$c: \underline{c}=(001):(\underline{004})$ & & - & 54.22 & 5426 \\
\hline
\end{tabular}

Fundort: Mina San Rafael, Sierra Gorda, Chile.

\section{Utahit.}

Der Utahit wurde im Jahre 1884 von Arzruni entdeckt und beschrieben 1). Das Mineral scheint seitdem nicht wieder beobachtet worden zu sein, wenigstens finden sich, soviel mir bekannt, abgesehen von der

1) Diese Zeitschr. 9, 558. 
zweifelhaften Notiz von F. A. Genth ${ }^{1}$ ), in der Literatur keine weiteren Mittheilungen darüber. Da somit bisher nur e in sicheres Vorkommen des Utahits bekannt war, dieses aber sowohl in Bezug auf krystallographische Ausbildung, wie auch als Analysenmaterial recht $\mathrm{zu}$ wünschen liess, so ist die Auffindung etwas besserer Krystalle immerhin erfreulich und man darf wohl hoffen, dass derselbe Fundort noch weiteres Material liefern wird.

Unter den von Herrn Hohmann früher hierher gesandten Proben chilenischen Minerals enthält nur eine den Utahit. Die braunen, noch nicht $1 \mathrm{~mm}$ grossen Kryställchen sitzen dichtgedrängt auf derbem gelblichbraunen Quarz. Einzelne Poren und kleinere Drusen des Gesteins sind gleichfalls mit Utahitkrystallen ausgekleidet. Bemerkenswerth ist das Zusammenvorkommen mit gediegenem Golde, das in kleinen Schüppchen zwischen den Krystallen des Utahits auftritt, zuweilen auch, als augenscheinlich jüngere Bildung, diese theilweise überzieht. Als Fundort giebt die Etikette an: Mina Santa Rosa, Guanaco, Taltal (Chile). Der Habitus der Krystalle ist pseudoregulär: man glaubt, die Combination Würfel mit Oktaëder, beides ungefähr im Gleichgewicht, zu sehen, während thatsächlich die rhomboëdrischen Formen

$$
\{10 \overline{1} 1\},\{02 \overline{2} 1\},\{0001\}
$$

vorliegen. Das Rhomboëder $\{02 \bar{Q} 1\}$ ist für den Utahit neu. Die Krystalle unterscheiden sich also sehr erheblich im Aussehen von den früher beschriebenen. Jenes waren winzige Schüppchen, fast mikroskopisch, mit vorherrschender Basis und untergeordneten Flächen des Rhomboëders $\{10 \overline{1} 1\}$. Entsprechend der bedeutenderen Grösse, und namentlich Dicke, sind die Krystalle des neuen Fundortes auch in der Farbe von den früheren erheblich verschieden; während jene sehr hell gelb erscheinen, mit fast metallischem Glanze auf der breiten Basis, sind die des neuen Vorkommens dunkel gelbbraun und glasglänzend. Ueberhaupt scheint zwischen beiden auf den ersten Blick wenig Aehnlichkeit zu bestehen, nicht mehr als etwa zwischen dem Eisenglanz von Elba und einem feinschuppigen Eisenglimmer. Dennoch scheinen mir die Ergebnisse der Messung wie der Analyse, ungeachtet mancher Unvollkommenheiten, jeden Zweifel an der Identität beider Exemplare auszuschliessen.

Trotz der anscheinend günstigeren Ausbildung der chilenischen Krystalle ist das Ergebniss der Messung wenig befriedigend.

\begin{tabular}{|c|c|c|c|}
\hline$(10 \pi$ & $\begin{array}{c}\text { Gemessen: } \\
52^{0} 18^{\prime}-53^{0} 12^{\prime}\end{array}$ & $\begin{array}{l}\text { Mittel: } \\
52^{\prime} 49^{\prime}\end{array}$ & $\begin{array}{c}\text { Berechnet } \\
-\end{array}$ \\
\hline$(02 \overline{2} 1):(00$ & $6743-6745$ & 6744 & $69^{\circ} \quad 3^{\prime}$ \\
\hline & $3-6022$ & $\begin{array}{ll}59 & 4.1 \\
& \text { (bez }\end{array}$ & $\begin{array}{ll}58 & 12 \\
59 & 31)\end{array}$ \\
\hline
\end{tabular}

1) Diese Zeitschr. 18, 590 . 
Der erste Winkel stimmt mit dem entsprechenden des Original-Utahits $\left.{ }^{1}\right)$ $(10 T 1):(0001)=5^{9} 45^{\prime}$ befriedigend überein. Wenn die Werthe der beiden anderen Winkel von den berechneten ziemlich abweichen, so ist daran in erster Linie die durch die Flächenbeschaffenheit bedingte Unvollkommenheit der Messung schuld, da schon die hier nur berücksichtigten besten $\mathbf{A b}$ lesungen um $1^{0}$ und mehr differiren.

Die Werthe für $(02 \overline{2} 1):(0001)=69^{\circ} 3^{\prime}$ und $(10 \overline{1} 1):(20 \overline{2} \bar{T})=58^{0} 12^{\prime}$ sind auf Grund der früheren Messung berechnet, welche für $a: c$ das Verhältniss $1: 1,1389$ ergab.

Legt man der Berechnung den Winkel $(09 \overline{2} 1):(0001)=67^{0} 44^{\prime} \mathrm{zu}$ Grunde (in dem alle Messungen am besten ühereinstimmen), so erhält man

$$
a: c=1: 1,0576
$$

und es ergiebt sich für (10T1):(0001) der Werth 50041', allerdings ziemlich abweichend von den Messungen (kleinster Werth $\left.{ }^{1}\right) \mathbf{5 2}^{0} \mathbf{2}^{\prime}$ ), dagegen für $(10 T 1):(20 \overline{2} T)$ in besserer Uebereinstimmung mit meinen Messungen $59^{0} 31^{\prime}$.

Es wird noch weiterer Untersuchungen an geeigneterem Materiale bedürfen, um zu entscheiden, welchen Winkelwerthen und welchem Axenverhältnisse also der Vorzug zu geben ist.

Das optische Verhalten des Original-Utabits schilderte $\Lambda \mathrm{rzruni}^{2}$ ) mit folgenden Worten: „Unter dem Mikroskope erkennt man fast bei allen Krystallen einen mehr oder minder scharf begrenzten, seinen Contouren nach mit den Umrissen des Krystalles concentrischen, dunkleren, braunen bis braunrothen Kern, während der breitere Rand die grellgelbe Farbe des bekannten Wulfenits von Utah besitzt. Diese Farbe entspricht der Reihe 6 $=$ Orange, zweiter Uebergang nach Gelb" der Radde'schen Scala und variirt je nach der Dicke der Kryställchen zwischen $6 \mathrm{r}$ und $6 \mathrm{u}$. Die Farbe des Kernes entspricht ungefähr derjenigen von $5 \mathbf{i}$ in derselben Scala. Der Kern dürfte als durch ausgeschiedenes Eisenozydhydrat gefärbt anzusehen sein, da er nicht mehr ganz durchsichtig ist und die einaxige Interferenzfigur nicht deutlich zeigt. e Vorher war gesagt worden, dass die basalen Täfelchen im convergenten Lichte vein scharfes, ungestörtes Interferenzbild einer einaxigen optisch negativen Substanz « zeigen, sowie dass - entsprechend dem hexagonalen Systeme - diese Täfelchen keinen Dichroïsmus aufweisen.

Diese Beschreibung passt fast genau auch auf die Krystalle aus Chile. Ihrer grösseren Dicke entsprechend sind hier die Farbentöne natürlich tiefer. Auch der dunkle Kern ist deutlich, oft schon mit der Lupe erkennbar. In einem Punkte gestattet das neue Material eine Ergänzung der früheren Beobachtungen. Das Mineral besitzt nämlich deutlichen Pleochroïsmus, wie man beim Durchsehen nach den grossen Flächen von $\{10 T 1\}$ erkennt; die
1) Arzruni l. c. 5 s̆9.
2) l. c. 
Farbe wechselt hier (parallel den Seiten der quadratischen Rhomboëderflächen) von ziemlich hellem Gelb (etwa 6o-p, Radde) zu einem tiefen Gelbbraun. Bei dem pseudoregulären Habitus der Krystalle liess sich nicht mit Sicherheit erkennen, welches die Richtung der Hauptaxe, bezw, die ihr entsprechende Farbe sei. Bei den früheren tafelförmigen Krystallen mit nur minimalen Seitenflächen hatte der Pleochroïsmus natürlich nicht festgestellt werden können. Als weitere Eigenthümlichkeit zeigen die neuen Krystalle auf der Basis im convergenten Lichte ein meist deutlich zweiaxiges Interferenzbild. Dass die Erscheinung jedoch als Anomalie zu betrachten ist, folgt nicht nur aus der rhomboëdrischen Gestalt, sondern auch noch mehr aus der Ungleichmässigkeit der Erscheinung selbst: nicht nur wechselt nämlich die Grösse des Axenwinkels von Ort zu Ort bis stellenweise fast zu völliger Einaxigkeit, sondern es ist auch nicht einmal die Lage der Axenebene constant. Anscheinend stellt diese Anomalie ein höheres Stadium der schon in der ersten Beschreibung des Utahits (s. oben) hervorgehobenen Erscheinung dar, dass der dunkle Kern die einaxige Interferenzfigur nicht deutlich zeigt«. Eine weitere interessante Beleuchtung erhält diese optische Eigenthümlichkeit des Utahits durch die ebendort ${ }^{1}$ ) mitgetheilte, beim Auflüsen in $\mathrm{HCl}$ gemachte Beobachtung, dass hierbei »das zwischen gekreuzten Nicols bis dahin dunkel erscheinende Blättchen, beim Drehen in seiner Ebene, je nach seiner Stellung abwechselnd hell und dunkel wird «, und dass man ferner im convergenten Lichte eine Dislocirung des bis dahin ungestörten

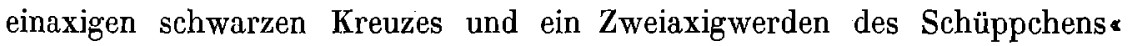
wahrnimmt.

Auch die neuen, von Natur anomal zweiaxigen Krystalle zeigen auf (0001) gesehen beim Drehen zwischen gekreuzten Nicols Aufhellung und werden nur felderweise dunkel. Ihr optisches Verhalten stimmt mit demjenigen der früher beobachteten Blättchen unter der Einwirkung von Salzsäure überein. Die im Anschlusse an diese Wahrnehmungen schon damals gemachte Bemerkung ${ }^{1}$ ), dass »optische Anomalien wohl nicht selten mit einer mehr oder minder weitgehenden chemischen Veränderung der Substanz in Zusammenhang zu bringen sind«, findet somit, für den Utahit wenigstens, eine neue Bestätigung.

\section{Brochantit.}

Die Analyse eines Brochantits aus Chile ist im chemischen Abschnitte mitgetheilt. Zu näherer mineralogischer Untersuchung gab die Stufe keinen Anlass.

Fundort: Mina Montecristo, Paposo, Chile.

1) 1. c. 560 . 
238 A. Arzruni $(t)$ und $\mathbf{K}$. Thaddéeff, vollendet und herausgeg. von A. Dannenberg.

\section{Wismuthcarbonat.}

Das neue Wismuthcarbonat, dem der Analyse und Berechnung von Herrn Thaddéeff zufolge vermuthlich die Formel $5 \mathrm{Bi}_{2} \mathrm{O}_{3} \cdot \mathrm{H}_{2} \mathrm{O} . \mathrm{CO}_{2}$ zukommt, tritt als traubiger Ueberzug von hellgelber Farbe auf, gebildet aus dichtgedrängten kugeligen Krystallaggregaten. Die Unterlage bilden Quarzkrystalle. Der Durchmesser der einzelnen kugeligen Krystallgruppen erreicht $1 \mathrm{~mm}$ oder etwas darüber.

Wird ein Theil eines solchen Aggregates unter das Mikroskop gebracht, so erkennt man darin fächerfürmige Verwachsungen kleiner Täfelchen von quadratischem Umrisse und sehr blassgelblicher Färbung oder auch fast farblos. Im convergenten Lichte sieht man in der Regel ein deutliches einaxiges Interferenzbild, zuweilen auch ein zweiaxiges Bild mit kleinem Axenwinkel, letzterer Fall stellt wohl eine Anomalie dar.

Das Mineral ist somit als dem quadratischem Systeme angehörig zu betrachten.

Fundort: Schneeberg in Sachsen.

\section{Chemische Untersuchungen von K. Thaddéeff.}

\section{Arzirunit.}

Die qualitative Untersuchung der weissen Masse, in welcher das zur Analyse verwandte Material von Arzrunit eingewachsen ist, ergab die Anwesenheit von $\mathrm{SiO}_{2}, \mathrm{HCl}, \mathrm{H}_{2} \mathrm{SO}_{4}, \mathrm{PbO}, \mathrm{CuO}, \mathrm{Fe}_{2} \mathrm{O}_{3}, \mathrm{ZnO}, \mathrm{CaO}$ (Spuren) und $\mathrm{H}_{2} \mathrm{O}$.

Da es nicht möglich war, zur Analyse genügendes Material an reinen Krystallen zu erhalten, musste ich einen Theil der Stufe zerkleinern, um zunächst die schwereren Bestandtheile - durch dreimalige Behandlung mit Methylenjodid - von Quarz zu reinigen. Aus diesem schweren Theile wurden mit der Lupe rein grünlich-blaue Stückchen ausgesucht, die zur Analyse verwendet wurden.

Aus der ersten Stufe erhielt ich im Ganzen ca. 0,2 g. Zur Controle wiederholte ich dasselbe Verfahren an einer kleineren Stufe; diese ergab mir ca. 0,19 g mehr grünlicher als blauer Substanz. Beide Proben wurden getrennt analysirt.

Das Mineral ist vor dem Löthrohre auf der Kohle leicht schmelzbar, dabei bräunt es sich und färbt die Flamme schön blau $\left(\mathrm{CuCl}_{2}\right)$; nach dem Schmelzen bleibt ein dunkelbrauner Regulus. Im Probirröhrchen erhitzt bräunt sich das Mineral ebenfalls, giebt Wasser ab, und schmilzt dann zu einer theils grünlichweissen, theils dunkelbraunen Masse. Beim Schmelzen 
mit Soda auf der Kohle erhält man einen Bleiregulus uud eine starke Heparreaction. Beim Auflösen in $\mathrm{HNO}_{3}$ hinterlässt das Mineral einen weissen Rückstand, die Lösung giebt mit $\mathrm{AgNO}_{3}$ deutliche Peaction auf $\mathrm{HCl}$.

Quantitative Analyse. Stufe I: 0,2 g abgewogen in Platinschale, mit Wasser gekocht, es fand keine Zersetzung statt; das Mineral behielt seine blave Farbe. Dann wurde $1 \mathrm{~g} \mathrm{Na}_{2} \mathrm{CO}_{3}$ dazugegeben, eine Stunde auf dem Wasserbade stehen gelassen und abfiltrirt. Diese Operation noch zweimal wiederholt, wobei die Lösung das letzte Mal die Nacht über auf dem Wasserbade stand.

Die Filtrate, welche $\mathrm{HCl}, \mathrm{B}_{2} \mathrm{SO}_{4}$, sowie Spuren von $\mathrm{Pb}$ und $\mathrm{Cu}$ erhielten, säuerte ich mit $\mathrm{HNO}_{3}$ an und fällte $\mathrm{HCl}$ mit $\mathrm{AgNO}_{3}$ (später als $\mathrm{Ag}$ gewogen), filtrirte und beseitigte das überschüssige $\mathrm{AgNO}_{3}$ mit $\mathrm{HCl}$. Nach dem Filtriren fällte ich $\mathrm{H}_{2} \mathrm{SO}_{4}$ mit $B a C l_{2}$. Das Filtrat wurde zur Trockne eingedampft und mit verdiunnter $\mathrm{HCl}$ aufgenommen, wobei sich die letzten Spuren von $\mathrm{BaSO}_{4}$ abschieden. In das Filtrat leitete ich unter Erwärmen $H_{2} S$ und vereinigte den hierbei entstandenen geringen Niederschlag von $P b S$ und $C u S$ nach dem Abfiltriren mit dem Hauptniederschlage (s. unten).

Der $\mathrm{BaSO}_{4}$-Niederschlag wurde nach Veraschen im Platintiegel mit $\mathrm{Na}_{2} \mathrm{CO}_{3}$ aufgeschlossen, in Wasser gelöst und die Lösung von $\mathrm{BaCO}_{3}$ abfiltrirt. Dás Filtrat säuerte ich mit $\mathrm{HCl}$ an, dampfte zur Trockne ein, löste abermals mit $\mathrm{HCl}$, filtrirte die vorhandenen Spuren von $\mathrm{SiO}_{2}$ ab und fällte von Neuem $\mathrm{H}_{2} \mathrm{SO}_{4}$ mit $\mathrm{BaCl}_{2}$.

Bestimmung der Basen. Der nach dreimaligem Kochen mit $\mathrm{Na}_{2} \mathrm{CO}_{3}$ verbliebene Rückstand wurde in $\mathrm{HNO}_{3}$ gelöst und von unlöslicher $\mathrm{SiO}_{2}$ abfiltrirt. Letztere wurde mit $\mathrm{HF}^{\prime}$ und $\mathrm{H}_{2} \mathrm{SO}_{4}$ auf Reinheil geprüft, hierbei im Platintlegel zurückgebliebene Spuren von Basen gelöst und die Lösung mit dem HauptGiltrate vereinigt.

In die stark verdünnte, kalte salpetersaure Lösung leitele ich $H_{2} S$ ein. Das ausgeschiedene $P b S_{2}$ und $C u S$ veraschte ich vorsichtig und löste danach in $\mathrm{HNO}_{3}$ auf. Diese Lösung dampfte ich unter Zusatz von $\mathrm{H}_{2} \mathrm{SO}_{4}$ ein und rauchte die überschüssige Säure ab; nach dem Abkühlen setzte ich noch verdünnte $\mathrm{H}_{2} \mathrm{SO}_{4}$ hinzu. Die Lösung wurde, nach Eindampfen auf dem Wasserbade, über der freien Flamme erhitzt, bis Dämpfe von $\mathrm{H}_{2} \mathrm{SO}_{4}$ aufstiegen, dann wieder abgekühlt, mit Wasser ver dünnt und absitzen gelassen.

Das ausgeschiedene $\mathrm{PbSO}_{4}$ wurde nun abfiltrirt, wobei ich zum Waschen zuerst verdünnte $\mathrm{H}_{2} \mathrm{SO}_{4}$, dann Alkohol benutzte.

Im Hauptfiltrate waren $\mathrm{Fe}_{2} \mathrm{O}_{3}, \mathrm{CaO}$ und $\mathrm{ZnO}$ geblieben. Ich bestimmte darin $\mathrm{Fe}_{2} \mathrm{O}_{3}$ und $\mathrm{CaO}$ nach den üblichen Methoden. Die Bestimmung des $\mathrm{ZnO}$ misslang bei dieser Probe.

Stufe II: abgewogen 0,1924 $\mathrm{g}$ in einen Porzellantiegel. Nach einstündigem Erwärmen im Trockenkasten bei $105^{\circ} \mathrm{C}$. war kein Verlust zu bemerken. Beim directen schwachen Erhitzen mit dem Gasbrenner im gut bedeckten Tiegel, unter Dunkelrothgluth, entwich Wasser. Nach dreistündigem Erhitzen war das Gewicht constant und die Masse wurde braun.

Weiter fübrte ich dann die Analyse wie bei Stufe I. Nur fällte ich diesmal, nachdem das Eisen ausgeschieden war, $Z n O$ aus essigsaurer Lösung bei Gegenwart von Natriumacetat mit $\mathrm{H}_{2} \mathrm{~S}$.

Die beiden Analysen ergaben:

Stufe I. Abgewogen 0,2 g. Gefunden: $\mathrm{SiO}_{2} 0,0272 \mathrm{~g}, \mathrm{Ol} 0,028774 \mathrm{~g}$ (berechnet ous $\mathrm{Ag} 0,0876 \mathrm{~g}), \mathrm{SO}_{3} 0,01613 \mathrm{~g}\left(\mathrm{BaSO}_{4} 0,0470 \mathrm{~g}\right), \mathrm{PbO} 0,06283 \mathrm{~g}\left(\mathrm{PbSO}_{4} 0,0854 \mathrm{~g}\right)$, $\mathrm{CuO} 0,04278 \mathrm{~g}\left(\mathrm{Cu}_{2} \mathrm{~S} 0,0428 \mathrm{~g}\right), \mathrm{Fe}_{2} \mathrm{O}_{3} 0,0014 \mathrm{~g}, \mathrm{CaO} 0,0034 \mathrm{~g}$. 
240 A. Arzruni ( $($ ) und K. Thaddéeff, vollendet und herausgeg. von A. Dannenberg.

Stufe II. Abgewogen 0,1924 g. Gefunden: $\mathrm{HH}_{2} \mathrm{O}$ (Glühverlust) $0,0212 \mathrm{~g} ; \mathrm{SiO}_{2}$

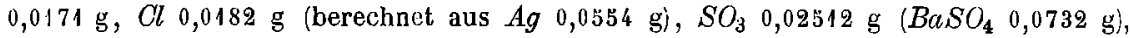

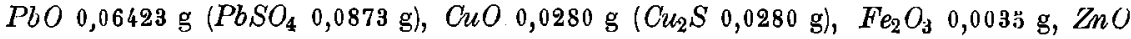
$0,007845 \mathrm{~g}(\mathrm{ZmS} 0,0094 \mathrm{~g}), \mathrm{CaO} 0,0036 \mathrm{~g}$.

Das giebt in Procenten:

$\begin{array}{lcr} & \text { Stufe I: } & \text { Stufe II: } \\ \mathrm{SiO}_{2} & 13,60 & 8,88 \\ \mathrm{SO}_{3} & 8,07 & 13,06 \\ \mathrm{Cl} & 14,39 & 9,46 \\ \mathrm{PbO} & 31,41 & 33,38 \\ \mathrm{CuO} & 21,39 & 14,54 \\ \mathrm{Fe}_{2} \mathrm{O}_{3} & 0,70 & 1,81 \\ \mathrm{CaO} & 1,70 & 1,87 \\ \mathrm{ZnO} & \text { nicht bestimmt } & 4,08 \\ \mathrm{H}_{2} \mathrm{O} & - & 11,01 \\ & & 98,09\end{array}$

Es ist gewiss eine schwierige Aufgabe, aus zwei so verschiedenen Analysen die richtige Formel des Minerals festzustellen, aber es ist doch möglich. Als Beimengungen können wir in der analytischen Substanz betrachten:

$\mathrm{SiO}_{2}$ (als Quarz), $\mathrm{Fe}_{2} \mathrm{O}_{3}$ (als $2 \mathrm{Fe}_{2} \mathrm{O}_{3} .3 \mathrm{H}_{2} \mathrm{O}$ ), $\mathrm{CaO}$ (als $\mathrm{CaSO}_{4}+2 \mathrm{H}_{2} \mathrm{O}$ ) und $\mathrm{ZnO}$ (als $\mathrm{ZnSO}_{4}+7 \mathrm{H}_{2} \mathrm{O}$ ). Nehmen wir die Molekularverhältnisse aus der Stufe II, so bekommen wir:

\begin{tabular}{|c|c|c|c|c|c|}
\hline & Mol.-Verh.: & $\begin{array}{c}2 \mathrm{Fe}_{2} \mathrm{O}_{3} \\
\mathbf{3} \mathrm{H}_{2} \mathrm{O}\end{array}$ & $\begin{array}{l}\mathrm{CaSO}_{4} . \\
2 \mathrm{H}_{2} \mathrm{O}\end{array}$ & $\begin{array}{c}\mathrm{ZnSO}_{4} . \\
6 \mathrm{H}_{2} \mathrm{O}\end{array}$ & Rest: \\
\hline $\mathrm{SiO}_{2}$ & 8,88 & 一 & - & - & 一 \\
\hline $\mathrm{SO}_{3}$ & $13,06: 79,86=0,163$ & - & $-0,033$ & $-0,050$ & 0,080 \\
\hline$C l$ & $9,46: \quad 35,37=0,267$ & 一 & 一 & - & 0,267 \\
\hline $\mathrm{PbO}$ & $33,38: 222,35=0,150$ & - & 一 & 一 & 0,150 \\
\hline $\mathrm{CuO}$ & $14,54: 79,14=0,183$ & 一 & 一 & 一 & 0,183 \\
\hline $\mathrm{Fe}_{2} \mathrm{O}_{3}$ & $1,81: 159,64=0,011$ & $-0,011$ & 一 & 一 & 0 \\
\hline $\mathrm{CaO}$ & $1,87: 55,87=0,033$ & 一 & $-0,033$ & 一 & 0 \\
\hline $\mathrm{ZnO}$ & $0,50: 80,84=0,050$ & - & 一 & $-0,050$ & 0 \\
\hline $\mathrm{H}_{2} \mathrm{O}$ & $11,01: 17,96=0,613$ & $-0,016$ & $-0,066$ & $-0,350$ & 0,181 \\
\hline
\end{tabular}

Die Löslichkeit der salzsauren Verbindung in verdünnter $H N O_{3}$, die vollständige Entfärbung des Minerals dabei, die schöne blaue Färbung der Löthrohrflamme, alles spricht dafür, dass hier Kupfer als eine salzsaure Verbindung und Blei als Sulfat vorhanden ist. Die Unlöslichkeit der Kupferverbindung in heissem Wasser zeigt, dass diese Verbindung eine basische ist. Auf Grund dieser Erwägungen gelangt man $\mathrm{zu}$ folgenden Molekularverhältnissen : 


\begin{tabular}{|c|c|c|c|c|c|}
\hline $\mathrm{PbO}$ & 0,150 & 1,87 & (2) & & PhSO PhO (Lanarkit) \\
\hline$S O_{:}$ & 0,080 & 1 & & & $\operatorname{POSU}_{4} \cdot \operatorname{PoU}$ (Landiki) \\
\hline $\mathrm{CuO}$ & 0,183 & 1 & & 4 & \\
\hline $\mathrm{Cl}$ & 0,267 & 1,46 & $(1, \breve{b})$ & 6 & $3\left(\mathrm{CuCl}_{2} \cdot \mathrm{H}_{2} \mathrm{O}\right)+\mathrm{Cu}(\mathrm{OH})_{2}$ \\
\hline $\mathrm{H}_{2} \mathrm{O}$ & 0,181 & 1 & & 4 & \\
\hline
\end{tabular}

Wie man sieht, ist also der Arzrunit nichts anderes als eine Doppelverbindung von dem basischen schwefelsauren Bleioxyd (Lanarkit $\left[\mathrm{Pb}_{2} \mathrm{O}\right] \mathrm{SO}_{4}$ ) und dem basischen Kupferchlorid. Da die weisse Masse, in welcher sich das Mineral befindet, hauptsächlich aus Lanarkit besteht und allmählich, ohne scharfe Grenzen, durch grünliche und grünlichblaue Färbungen in die blaue Substanz übergeht, so war es mir kaum möglich, diese letztere frei von Lanarkit zu bekommen. Daher ist es schwer, aus der Analyse der Stufe II, bei welcher das Material nicht so rein war wie bei Stufe I, das Verhältniss festzustellen, in welchem Bleisulfat und Kupferchlorid zu einander stehen. Dass der Arzrunit ein Doppelsalz ist, zeigten deutlich die qualitativen Proben mit ausgesuchten reinen, schön blauen Kryställchen. Die Kryställchen von verschiedenen Stufen zeigten bei der qualitativen Untersuchung immer dieselbe Zusammensetzung: $\mathrm{PbO}, \mathrm{CuO}, \mathrm{SO}_{3}, \mathrm{HCl}$ und $\mathrm{H}_{2} \mathrm{O}$. Da die erste Stufe besseres Material lieferte, so lässt sich vielleicht aus dieser Analyse ein richtigeres Verhältniss zwischen $\mathrm{PbSO}_{4} \cdot \mathrm{PbO}$ und $3\left(\mathrm{CuCl}_{2} \mathrm{H}_{2} \mathrm{O}\right)+\mathrm{Cu}(\mathrm{OH})_{2}$ ableiten. Berechnen wir diese Analyse ebenso wie die der zweiten Stufe, so bekommen wir:

\begin{tabular}{lrrcccc} 
& \multicolumn{1}{c}{$\%$} & & Mol.-Verh.: & $2 \mathrm{Fe}_{2} \mathrm{O}_{3} .3 \mathrm{H}_{2} \mathrm{O}$ & $\mathrm{CaSO}_{4} .2 \mathrm{H}_{2} \mathrm{O}$ & Rest: \\
$\mathrm{SiO}_{2}$ & 13,60 & & - & - & - & - \\
$\mathrm{SO}_{3}$ & $8,07:$ & 79,86 & 0,1010 & - & $-0,0304$ & 0,0706 \\
$\mathrm{Cl}$ & $14,39:$ & 35,37 & 0,4068 & - & - & 0,4068 \\
$\mathrm{PbO}$ & $31,41: 222,35$ & $0,14.12$ & - & - & 0,1412 \\
$\mathrm{CuO}$ & $21,39:$ & 79,14 & 0,2702 & - & - & 0,2702 \\
$\mathrm{Fe}_{2} \mathrm{O}_{3}$ & $0,70: 159,64$ & 0,0044 & $-0,0044$ & - & 0 \\
$\mathrm{CaO}$ & $1,70:$ & 55,87 & 0,0304 & - & $-0,0304$ & 0
\end{tabular}

Bei Zusammenstellung erhalten wir wieder dieselben Verhältnisse von $\mathrm{PbO}: \mathrm{SO}_{3}(2: 1)$ und $\mathrm{CuO}: \mathrm{Cl}(1: 1,5)$ wie bei der zweiten Stufe, nämlich:

\begin{tabular}{|c|c|c|c|}
\hline $\mathrm{PbO}$ & 0,1412 & (2) & \multirow{2}{*}{$\mathrm{PbSO}_{4} \cdot \mathrm{PbO}$} \\
\hline $\mathrm{SO}_{3}$ & 0,0706 & (1) & \\
\hline $\mathrm{CuO}$ & 0,2702 & (1) & \multirow{2}{*}{$1 \mathrm{CuO} \cdot 1,5 \mathrm{Cl}$} \\
\hline$C l$ & 040 & 15 & \\
\hline
\end{tabular}

Da das Verhältniss von $\mathrm{PbO}$ zu $\mathrm{CuO}$ 0,1412:0,2702 =1:1,907 oder fast 1:2 ist, so müssen wir dementsprechend die Verhältnisse in Kupferchlorid vierfach nehmen, um die vollständige Formel des Arzrunits zu bekommen, also: 
242 A. Arzruni ( + ) und K. Thaddeéff, vollendet und herausgeg. von A. Dannenberg.

$$
\left.\begin{array}{ll}
\mathrm{PbO} & 2 \\
\mathrm{SO}_{3} & 1 \\
\mathrm{CuO} & 4 \\
\mathrm{Cl} & 6
\end{array}\right\} \mathrm{PbSO}_{4} \cdot \mathrm{PbO} \cdot 3 \mathrm{CuCl}_{2} \cdot \mathrm{CuO}
$$

Nehmen wir nun die Wasserbestimmung aus der Analyse der Stufe II hinzu, so lautet nunmehr die Formel:

$$
\mathrm{PbSO}_{4} \cdot \mathrm{PbO}+3\left(\mathrm{CuCl}_{2} \cdot \mathrm{H}_{2} \mathrm{O}\right)+\mathrm{Cu}(\mathrm{OH})_{2} \text {. }
$$

Beide Analysen zeigen einen Ueberschuss an $\mathrm{PbSO}_{4} . \mathrm{PbO}$, der sich aber leicht erklärt durch die Schwierigkeit der Trennung von Lanarkit $\left(\mathrm{PbSO}_{4}, \mathrm{PbO}\right)$.

\section{Stelznerit.}

Die Substanz bei $160^{\circ}-170^{\circ}$ getrocknet erlitt keinen Verlust.

Specifisches Gewicht in Wasser (worin das Mineral nicht löslich) mit dem Pyknometer bestimmt:

1) 1,74.93 g gaben als Mittel aus drei Bestimmungen

$$
\text { bei } 16,5 \mathrm{C} .+4^{0} \mathrm{C} \text {. } 3,878
$$

2) 1,7330 g gaben als Mittel aus zwei Bestimmungen

$$
\text { in gleicher Weise } \quad 3,890
$$

also aus fünf Bestimmungen im Mittel $D=3,884$.

Analys :

I) $0,5 \mathrm{~g}$ gaben in $\mathrm{HCl}$ unlöslichen Rückstand $0,0024 \mathrm{~g}=0,48 \%$

$$
\begin{aligned}
& C u, S \quad 0,3355=C u O \quad 0,3350 \mathrm{~g}=67,08 * \\
& \mathrm{Fe}_{2} \mathrm{O}_{3} \quad 0,0017 \mathrm{~g}=0,34 \text {, }
\end{aligned}
$$$$
\mathrm{CaO} 0,0003 \mathrm{~g}=0,06 \text {, }
$$

\begin{tabular}{|c|c|c|c|c|c|c|c|}
\hline & I. & II. & III. & Mittel: & Mol.-Verb.: & & \\
\hline $\mathrm{CuO}$ & 67,08 & - & 一 & $67,08: 79,14$ & 0,847 & 3,02 & (3) \\
\hline $\mathrm{SO}_{3}$ & 一 & 22,40 & - & $22,40: 79,86$ & 0,280 & 4 & \\
\hline $\mathrm{H}_{2} \mathrm{O}$ & - & 一 & 10,22 & $10,22: 17,96$ & 0,569 & 2,02 & (2) \\
\hline $\mathrm{Fe}_{2} \mathrm{O}_{3}$ & 0,34 & 一 & - & 0,34 & & & \\
\hline $\mathrm{CaO}$ & 0,06 & - & - & 0,06 & & & \\
\hline Rückstand & 0,48 & 0,40 & - & 0,44 & & & \\
\hline & & & & $10 \overline{0,54}$ & & & \\
\hline
\end{tabular}
II) $0,5 \mathrm{~g}$ gaben unlöslichen Rückstand $0,0020 \mathrm{~g}=0,40$

$$
\mathrm{BaSO}_{4} \mathbf{0 , 3 2 6 3}=\mathrm{SO}_{3} \quad 0,1120 \mathrm{~g}=22,40 \text {, }
$$

III) $0,5 \mathrm{~g}$ gaben mit $3 \mathrm{~g} \mathrm{KNaCO}$ im Porzellanrohre geschmolzen

$$
\text { im } \mathrm{CO}_{2} \text {-Strome: } \quad \mathrm{H}_{2} \mathrm{O} \quad 0,0511 \mathrm{~g}=10,220 \%
$$

Das Molekularverhältniss führt zu der Formel:

$$
3 \mathrm{CuO} .1 \mathrm{SO}_{3} \cdot 2 \mathrm{H}_{2} \mathrm{O} \text { oder } \mathrm{CuSO}_{4} \cdot 2 \mathrm{Cu}(\mathrm{OH})_{2} \text {. }
$$

Ziehen wir $\mathrm{Fe}_{2} \mathrm{O}_{3}, \mathrm{CaO}$ und den Rückstand $(0,34+0,06+0,44=)$ $0,84 \%$ von $100,5 \%$ ab und rechnen den Rest auf $100 \mathrm{um}$, so bekommen wir: 


$\begin{array}{lccr} & \text { Gefunden: } & \begin{array}{c}\text { Berechnet für } \\ \mathrm{CuSO} \cdot 2 \mathrm{Cu}(\mathrm{OH})_{2}\end{array} & \text { Differenz : } \\ \mathrm{CuO} & 67,28 & 67,22 & +0,06 \\ \mathrm{SO}_{3} & 22,46 & 22,61 & -0,15 \\ \mathrm{H}_{2} \mathrm{O} & \frac{10,25}{99,99} & \frac{10,17}{1.00,00} & +0,08\end{array}$

Die erste Analyse des Stelznerits war von Herrn Bergbaubeflissenen Schröcker ausgeführt worden. Er fand:

I) Abgewogen 1 g. Gangart: 0,0142; $\mathrm{Cu}_{2} \mathrm{~S}$ 0,6420=CuO 0,6417, $\mathrm{Fe}_{2} \mathrm{O}_{3}$ 0,0114, $\mathrm{CaO} 0,0057$.

II) Abgewogen $1 \mathrm{~g}$. $\mathrm{Cu} \mathrm{S}_{0,6386}=\mathrm{CuO} 0,6383$.

III) Abgewogen 0,4379. $\mathrm{BaSO}_{4} 0,2832=\mathrm{SO}_{3} 0,0972$.

1V) Abgewogen 1,0572 im Platinschiffchen gab bei $100^{\circ}$ getrocknet Verlust: $\mathrm{H}_{2} \mathrm{O}$ $0,0035 \mathrm{~g}$. Nach Aufschliessen mit $\mathrm{KNaCO}_{3}(4 \mathrm{~g})$ in der Porzellanröhre nach der Methode von $\mathrm{Sipöcz}$ gefunden $\mathrm{H}_{2} \mathrm{O} 0,1096 \mathrm{~g}$.

\begin{tabular}{lcccc|cc} 
& I. & II. & III. & IV. & Summe: & Mol.-Verh.: \\
$\mathrm{CuO}$ & 64,17 & 63,86 & - & - & $64,01: 79,14$ & 0,808 \\
$\mathrm{SO}_{3}$ & - & - & 22,19 & - & $22,19: 79,68$ & 0,277 \\
$\mathrm{H}_{2} \mathrm{O}$ & - & - & - & 10,37 & $10,37: 17,96$ & 0,577 \\
$\mathrm{Fe}_{2} \mathrm{O}_{3}$ & 1,14 & - & - & - & 1,14 & \\
$\mathrm{CaO}$ & 0,57 & - & - & - & $0,57: 55,87$ & 0,010 \\
Gangart & 1,42 & - & - & - & 1,42 & \\
Feuchtigkeit & - & - & - & 0,33 & $\frac{0,33}{100,03}$ &
\end{tabular}

Zieht man $\mathrm{CaO}$ als $\mathrm{CaSO}_{4} \cdot 2 \mathrm{H}_{2} \mathrm{O}$ ab, so wird das Molekularverhältniss :

$\left.\begin{array}{ccccc}\mathrm{CuO} & 0,808 & - & 0,808 & 3,02 \\ \mathrm{SO}_{3} & 0,277 & -0,010 & 0,267 & 1 \\ \mathrm{H}_{2} \mathrm{O} & 0,577 & -0,020 & 0,557 & 2,08 \\ \mathrm{CaO} & 0,010 & -0,010 & & \end{array}\right\} 3 \mathrm{CuO} \cdot 1 \mathrm{SO}_{3} \cdot 2 \mathrm{H}_{2} \mathrm{O}$

woraus gleichfalls die Formel $\mathrm{CuSO}_{4} \cdot 2 \mathrm{Cu}(\mathrm{OH})_{2}$ folgt.

\section{Rafaëlit.}

Von dem geringen zur Untersuchung verfügbaren Material konnte nur eine qualitative Analyse ausgeführt werden. Auf der Kohle vor dem Löthrohre schmilzt das Mineral, giebt $\mathrm{PbO}$-Beschlag und wird zum Bleiregulus reducirt. Ebenso giebt es mit Soda auf der Kohle einen Bleiregulus. Im Probirröhrchen erhitzt schmilzt es zu einer weissen Masse. In $\mathrm{HNO}_{3}$ schwer löslich; die Lösung giebt mit $\mathrm{AgNO}_{3}$ deutliche Reaction auf $\mathrm{HCl}$.

Allem Anscheine nach liegt ein Bleioxychlorid vor. 
244 A. Arzruni (t) und K. Thaddeéff, vollendet und herausgeg. von A. Danuenberg.

\section{Basisches Eisenoxydsulfat (Utahit).}

Die qualitative Analyse zeigte die Anwesenheit von $\mathrm{Fe}_{2} \mathrm{O}_{3}, \mathrm{SO}_{3}$ und $\mathrm{H}_{2} \mathrm{O}$. Die Probe auf $\mathrm{As}$ gab ein negatives Resultat.

Quantitative Untersuchung. Abgewogen $0,0229 \mathrm{~g}$. Nach dem Auflösen in $\mathrm{HCl}$ blieb ein unlöslicher Rückstand von $0,0005 \mathrm{~g}=2,19 \%$. Im Filtrat schlug ich $\mathrm{Fe}_{2} \mathrm{O}_{3}$ mit Ammoniak nieder: gefunden $0,0134 \mathrm{~g}=\mathbf{3} 8,51 \% \mathrm{Fe}_{2} \mathrm{O}_{3}$. Dem Filtrat nach dem Ansäuern mit $\mathrm{HCl}, \mathrm{BaCl}_{2}$ zugesetzt, die Nacht über warm stehen gelassen, nach dem Abkühlen filtrirt ergab: $0,0179 \mathrm{~g} \mathrm{BaSO}_{4}$ entsprechend $0,006143 \mathrm{~g} \mathrm{SO}_{3}$ oder 26,83\%. Also im Ganzen gefunden:

$\begin{array}{llll}\text { Rückstand: } & 2,19 \% & \text { Mol. -Verh.: } & \\ \mathrm{Fe}_{2} \mathrm{O}_{3} & 58,51: 159,64=0,366 & 1,09 \\ \mathrm{SO}_{3} & \mathbf{2 6 , 8 3}: 79,86=0,336 & 1\end{array}$

Die Zusammensetzung stimmt fast genau überein mit der des Utahits, in welchem Damour fand:

$$
\begin{array}{lr}
\mathrm{Fe}_{2} \mathrm{O}_{3} & 58,82 \\
\mathrm{SO}_{3} & 28,4.5 \\
\mathrm{As}_{2} \mathrm{O}_{5} & 3,19 \\
\mathrm{H}_{2} \mathrm{O} & 9,35
\end{array}
$$

was nach Abrechnung des $\mathrm{As}_{2} \mathrm{O}_{5}$ als Skorodit $\left(\mathrm{Fe}_{2} \mathrm{As}_{2} \mathrm{O}_{8} \cdot 4 \mathrm{H}_{2} \mathrm{O}\right)$ zur Formel $3 \mathrm{Fe}_{2} \mathrm{O}_{3} \cdot 3 \mathrm{SO}_{3} \cdot 4 \mathrm{H}_{2} \mathrm{O}$ führt.

Der geringe Ueberschuss an $\mathrm{Fe}_{2} \mathrm{O}_{3}$ gegenüber dem Verhältniss $\mathrm{Fe}_{2} \mathrm{O}_{3}$ : $S O_{3}=1: 1$, den ich gefunden, lässt sich erklären durch beigemischten Limonit, den man deutlich zwischen den Kryställchen des untersuchten Minerals erkennt. Es ist auffallend, dass meine $\mathrm{Fe}_{2} \mathrm{O}_{3 j}$-Bestimmung beinahe dieselben Zahlen ergiebt, wie bei Damour's Analyse des Utahits, dass dagegen die $\mathrm{SO}_{3}$-Bestimmung, bei der ich grössere Genauigkeit erwarten konnte, um $1,62 \%$ abweicht.

So gewagt es erscheinen mag, auf Grund einer mit nur 0,0229 g Substanz ausgeführten Analyse eine Formel berechnen zu wollen, müchte ich doch einige Betrachtungen hierüber nicht unterlassen.

Aus der Differenz von $100 \%$ berechnet sich der $\mathrm{H}_{2} \mathrm{O}$-Gehalt zu $12,47 \%$. Zieht man weiter den Ueberschuss an Eisen als $2 \mathrm{Fe}_{2} \mathrm{O}_{3} .3 \mathrm{H}_{2} \mathrm{O}$ ab, so bekommt man folgende Verhältnisse:

$\begin{array}{lrrrrl} & \% & & \text { Mol.-Verh.: } & 2 \mathrm{Fe}_{2} \mathrm{O}_{3} .3 \mathrm{H}_{2} \mathrm{O} & \text { Rest: } \\ \text { Rückstand } & 2,19: & & & \\ \mathrm{Fe}_{2} \mathrm{O}_{3} & 58,51: 159,64=0,366 & -0,030 & 0,336 & 1 \\ \mathrm{SO}_{3} & 26,83: & 79,86=0,336 & - & 0,336 & 1 \\ \mathrm{H}_{2} \mathrm{O} & 12,47: & 17,96=0,694 & -0,045 & 0,649 & 1,93\end{array}$


Danach wäre die Formel: $\mathrm{Fe}_{2} \mathrm{O}_{3} \cdot \mathrm{SO}_{3} \cdot 2 \mathrm{H}_{2} \mathrm{O}$, was wieder zu der ganz einfachen Constitutionsformel führt:

$$
F e=\begin{aligned}
& O H \\
& O H \\
& S O_{4} \\
& O H \\
& O H
\end{aligned}
$$

welches Salz schon längst von Soubeiran ${ }^{1}$ ) als rothgelbes Pulver künstlich dargestellt ist.

Von Damour's Analyse des Utahits unterscheidet sich die gegenwärtige fast nur durch den etwas höheren Wassergehalt. Berücksichtigt man die Schwierigkeiten der Untersuchung: unreine Substanz in dem einen Falle, Bestimmung des Wassers aus der Differenz bei minimaler Menge und ebenfalls nicht ganz reiner Substanz im anderen Falle, so wird man kaum geneigt sein, aus diesen geringen Abweichungen auf eine wesentlich chemische Verschiedenheit der beiden Substanzen zu schliessen. Welcher der beiden sich danach für den Utahit ergebenden Formeln der Vorzug gebührt, der Damour'schen oder der hier abgeleiteten, lässt sich einstweilen nicht entscheiden. Die Einfachheit der sich aus letzterer ergebenden Constitutionsformel möchte wohl zu deren Gunsten sprechen.

\section{Brochantit.}

Beim Trocknen der Substanz bei $160^{\circ}-170^{\circ}$ fand kein Verlust statt.

I) Abgewogen $1 \mathrm{~g}$ gab in $\mathrm{HCl}$ unlöslichen Rückstand: $0,0024 \mathrm{~g}=0,240 \%$, $\mathrm{C}_{\imath_{2}} S 0,688 \mathrm{j} \mathrm{g}$ entspr. $\mathrm{CuO}: 0,6882 \mathrm{~g}=68,82$ -

$$
\begin{array}{r}
\mathrm{Fe}_{2} \mathrm{O}_{3}: 0,0207 \mathrm{~g}=2,07- \\
\mathrm{CaO}: 0,0005 \mathrm{~g}=0,05-
\end{array}
$$

II) Abgewogen $1 \mathrm{~g}$ gab Rückstand, nnlöslich in $\mathrm{HCl}$ : $\quad 0,0022 \mathrm{~g}=0,22-$

$$
B a S O_{4}: 0,5060 \mathrm{~g} \text { entspr. } S \dot{O}_{3}: 0,1737 \mathrm{~g}=17,37 \text {. }
$$

III) Abgewogen 0,5 g, mit $\mathrm{KNaCO}$ aufgeschlossen im Porzellanrohre im $\mathrm{CO}_{2}$ Strome? gaben $\mathrm{H}_{2} \mathrm{O}: \quad 0,0581 \mathrm{~g}=11,62 \%$.

\begin{tabular}{|c|c|c|c|c|c|c|c|}
\hline $\mathrm{CuO}$ & $68,8 \mathcal{2}$ & - & 一 & 一 & $68,82: 79,14$ & $=0,864$ & 4 \\
\hline $\mathrm{SO}_{3}$ & - & 17,37 & - & - & $17,37: 79,86$ & $=0,217$ & 1 \\
\hline $\mathrm{H}_{2} \mathrm{O}$ & - & - & 11,62 & 11,41 & $11,51: 17,96$ & $=0,641$ & 2,95 \\
\hline $\mathrm{Fe}_{2} \mathrm{O}_{3}$ & 2,07 & - & 一 & 一 & 2,07 & & \\
\hline $\mathrm{CaO}$ & 0,05 & 一 & 一 & - & 0,05 & & \\
\hline Rückst. & 0,24 & 0,22 & - & - & $\frac{0,23}{0005}$ & & \\
\hline
\end{tabular}

IV) $1 \mathrm{~g}$ Substanz mit $6 \mathrm{~g} K N a \mathrm{CO}_{\downarrow}$ in gleicher Weise behandelt gab $\mathrm{H}_{2} \mathrm{O}$ :

$$
\text { Zusammenstellung. } \quad 0,1141 \mathrm{~g}=11,41 \% \text {. }
$$

I. II. III. IV. Mittel: Mol. -Verh. :

1) Graham-Otto's Lehrbuch d. anorg. Chem. 1886, 5. Aufl., 2, IV. Abth., 1. Hälfte, 621.

2) Vergl, diese Zeitschr. 28, 272; Analyse des Sulfoborits. 
246 A. Arzruni ( $t$ ) und $\mathbf{K}$. Thaddeeff, vollendet und herausgeg. von A. Dannenberg.

Zichen wir $\mathrm{Fe}_{2} \mathrm{O}_{3}, \mathrm{CaO}$ und den Rückstand $(2,07+0,05+0,23=)$ $2,35 \%$ von $100,05 \%$ ab und berechnen den Rest auf 100 , so erhalten wir:

$\begin{array}{lccc} & \text { Gefunden: } & \begin{array}{c}\text { Berechnet für } \\ \mathrm{CuSO} \cdot 3 \mathrm{Cu}(\mathrm{OH})_{2}\end{array} & \text { Differenz: } \\ \mathrm{CuO} & 70,44 & 70,30 & +0,14 \\ \mathrm{SO}_{3} & 17,77 & 17,73 & +0,04 \\ \mathrm{H}_{2} \mathrm{O} & 11,78 & 11,96 & -0,18\end{array}$

\section{Basisches Wismuthcarbonat.}

Die hellgelbe, pulverige Substanz wurde mit Methylenjodid von Quarz gercinigt und dies drei Mal wiederholt; trotzdem enthielt die Substanz, wie die $\Lambda$ nalyse zeigte, noch bis $8 \%$ Quarz.

Wenn wir annehmen, dass die Substanz ein specifisches Gewicht nahe dem des Bismutits (bis 6,3) hat, während das des Quarzes 2,65 ist, sowie das der angewandten Trennungsflüssigkeit 3,2, so ist leicht verständlich, dass kleine Mengen anhängenden Wismuthsalzes Theilchen von Quarz mit hinabziehen.

Die qualitative Analyse zeigte die Anwesenheit von $\mathrm{Bi}_{2} \mathrm{O}_{3}, \mathrm{CO}_{2}$ und $\mathrm{H}_{2} \mathrm{O}$ als Haupthestandtheile.

Die erste Analyse führte ich qualitativ-quantitativ: nach Bestimmung des Glühverlustes löste ich die Substanz in $\mathrm{HNO}_{3}$, filtrirte von dem unlöslichen Rückstande (Quarz) ab und schlug Wismuth, sammt Spuren von Kupfer durch $H_{2} S$ nieder. Im Filtrate hiervon bestimmte ich $\mathrm{Fe}_{2} \mathrm{O}_{3}$ und $\mathrm{CaO}$ nach den üblichen Methoden.

Den Niederschlag von Schwefelwismuth (mit Spuren von Kupfer) löste ich in heisser $\mathrm{HNO}_{3}$ auf und dampfte - jedes Mal mit Zusatz von Wasser -- dreimal zur Trockne ein.

Den völlig trockenen Rückstand löste ich kalt in einer Lösung von $\mathrm{NH}_{4} \mathrm{NO}_{3}$ ( $1: 500 \quad B_{2} O$ ), filtrirte ab und wusch mit derselben Lösung aus. In der Lösung waren nur unbestimmbare Spuren von Kupfer geblieben.

In der zweiten Analyse bestimmte ich nur den Rüickstand und $\mathrm{CO}_{2}$.

Analyse I: 0,1 g Substanz gaben Glühverlust $0,0050 \mathrm{~g}, \mathrm{Bi}_{2} \mathrm{O}_{3} 0,0819 \mathrm{~g}, \mathrm{Fe}_{2} \mathrm{O}_{3}$ $0,0015 \mathrm{~g}, \mathrm{CaO} 0,0032 \mathrm{~g}$.

Analyse II: $0,3169 \mathrm{~g}$ gaben $0,0430 \mathrm{~g} \mathrm{CO}_{2}$ und 0,0264 g Rückstand.

\begin{tabular}{lrcrcc} 
& I. & II. & \multicolumn{1}{c}{ Summe: } & Mol. -Verh.: \\
$\mathrm{Bi}_{2} \mathrm{O}_{3}$ & $\mathbf{8 1 , 9 0}$ & - & $81,90: 464,68$ & 0,174 \\
$\mathrm{CaO}$ & 3,20 & - & $3,20:$ & 55,87 & 0,085 \\
$\mathrm{Fe}_{2} \mathrm{O}_{3}$ & 1,50 & - & $1,50: 159,64$ & 0,009 \\
$\mathrm{CO}_{2}$ & & 4,10 & $4,10:$ & 43,89 & 0,093 \\
$\mathrm{H}_{2} \mathrm{O}$ & 5,00 & - & $0,90:$ & 17,96 & 0,050 \\
Rückstand & - & $\mathbf{8 , 3 3}$ & $\mathbf{8 , 3 3}$ & &
\end{tabular}

Rechnen wir $\mathrm{CaO}$ (als $\mathrm{CaCO}_{3}$ ) und $\mathrm{Fe}_{2} \mathrm{O}_{3}$ (als $2 \mathrm{Fe}_{2} \mathrm{O}_{\mathbf{3}} \cdot 3 \mathrm{H}_{2} \mathrm{O}$ ) ab, so bekommen wir folgende Verhältnisse: 


$\begin{array}{llll}\mathrm{Bi}_{2} \mathrm{O}_{3} & 0,1 \% 4 & 4,97 & \text { (5) } \\ \mathrm{CO}_{2} & 0,035 & 1 & \\ \mathrm{H}_{2} \mathrm{O} & 0,037 & 1 & \end{array}$

also $5 \mathrm{Bi}_{2} \mathrm{O}_{3} . \mathrm{H}_{2} \mathrm{O} . \mathrm{CO}_{2}$, was in Procenten ergiebt: $\mathrm{Bi}_{2} \mathrm{O}_{3} 81,9, \mathrm{CO}_{2}$ 1, $\mathrm{H}_{2} \mathrm{O} \quad 0,66$ oder auf 100 berechnet:

\begin{tabular}{|c|c|c|c|}
\hline & & $\begin{array}{c}\text { Berechnet für } \\
5 \mathrm{Bi}_{2} \mathrm{O}_{3} . \mathrm{H}_{2} \mathrm{O} . \mathrm{CO}_{2}\end{array}$ & Differenz : \\
\hline $\mathrm{Bi}_{2} \mathrm{O}_{3}$ & 97,38 & 97,40 & $-0,02$ \\
\hline $\mathrm{CO}_{2}$ & 1,83 & 1,84 & $-0,01$ \\
\hline \multirow[t]{2}{*}{$H_{2} \mathrm{O}$} & 0,78 & 0,75 & $+0,03$ \\
\hline & 99,99 & 99,99 & \\
\hline
\end{tabular}

Es wäre voreilig, auf Grund dieser Analysen allein dieses Mineral als neu $\mathrm{zu}$ betrachten, und doch wird man eine solche Uebereinstimmung zwischen Analyse und Berechnung kaum einem Zufalle zuschreiben wollen.

Bis jetzt kennen wir in der Natur nur zwei ähnliche Verbindungen:

$\begin{array}{ll}\text { Bismutit } & 3 \mathrm{Bi}_{2} \mathrm{O}_{3} \cdot \mathrm{H}_{2} \mathrm{O} \cdot \mathrm{CO}_{2} \\ \text { Wismuthspath } & 4 \mathrm{Bi}_{2} \mathrm{O}_{3} \cdot 4 \mathrm{H}_{2} \mathrm{O} \cdot 3 \mathrm{CO}_{2} .\end{array}$ und

Das künstlich dargestellte Wismuthcarbonat hat nach Karl Seubert und M. Elten ${ }^{1)}$ die Zusammensetzung:

$(\mathrm{BiO})_{2} \mathrm{CO}_{3} \cdot \frac{1}{2} \mathrm{H}_{2} \mathrm{O}$ oder $\mathrm{Bi}_{2} \mathrm{O}_{3} \cdot \mathrm{CO}_{2} \cdot \frac{1}{2} \mathrm{H}_{2} \mathrm{O}$ (nach der Analyse ist der $\mathrm{H}_{2} \mathrm{O}$-Gehalt noch geringer).

Es ist leider so wenig bekannt über die kohlensauren Salze des Wismuthoxyds, dass es gegenwärtig noch vollständig unmöglich ist, sichere Schlüsse betreffs solcher Verbindungen zu ziehen. Es könnte sein, dass bei näherer Untersuchnng alle vorher erwähnten Salze als nichts anderes erkannt werden als Mischungen von $\mathrm{Bi}_{2} \mathrm{O}_{3}$ mit der Verbindung $\mathrm{Bi}_{2} \mathrm{O}_{\jmath} . \mathrm{H}_{2} \mathrm{O} . \mathrm{CO}_{2}$. Die Constitution der letzteren erklärt sich leicht aus der. Formel der Kohlensäure:

$$
\mathrm{H}_{2} \mathrm{CO}_{3}=\stackrel{\mathrm{H}}{\mathrm{H}}>\mathrm{CO}_{3} \text { giebt } \underset{\mathrm{BiO}}{\mathrm{Bi}(\mathrm{OH})_{2}}>\mathrm{CO}_{3} .
$$

Aehnliche Verbindungen haben wir z. B. bei den schwefelsauren Salzen ${ }^{2}$ ): $\mathrm{Bi}_{2} \mathrm{O}_{3} . \mathrm{SO}_{3} \cdot \mathrm{H}_{2} \mathrm{O}$ und $3 \mathrm{Bi}_{2} \mathrm{O}_{3} \cdot 2 \mathrm{SO}_{3} \cdot 2 \mathrm{H}_{2} \mathrm{O}$, wobei in den kohlensauren wie in den schwefelsauren Salzen das Verhältniss von $\mathrm{H}_{2} \mathrm{O}$ zur Säure gleich $1: 1$ ist.

1) Zeitschr. f. anorg. Chem. $1893,76$.

2) Ladenburg, Handwörterbuch der Chemie 13, 228. 